This is a post-peer-review, pre-copyedit version of a chapter published in Yan Q. (eds) Psychoneuroimmunology. Methods in Molecular Biology, vol 1781. Humana Press, New York, NY. The final authenticated version is available online at: https://doi.org/10.1007/978-1-4939-7828-1_16

\title{
The Vaccination Model in Psychoneuroimmunology Research: A Review
}

Running head: Vaccine research in PNI

Anna C. Whittaker, PhD CPsychol FBPsS

School of Sport, Exercise \& Rehabilitation Sciences, University of Birmingham, Edgbaston,

Birmingham, B15 2TT. Phone: 0121414 4398. Fax: 0121414 4121. A.C.Phillips@ bham.ac.uk 


\section{Summary}

This chapter explores the reasoning behind using the vaccination model to examine the influence of psychosocial factors on immunity. It then briefly discusses the mechanics of the vaccination response and the protocols used in Psychoneuroimmunology vaccine research, before giving examples from the research literature of the studies examining relationships such as the association between stress and the vaccination response. It also explores the ways the vaccination model can be used to answer key questions in Psychoneuroimmunology, such as: does it matter when stressful life events occur relative to when the vaccine is received?' 'what are the effects of prior exposure to the antigen?' and 'do other psychosocial factors influence vaccine response besides stress?' Finally, it briefly considers the mechanisms underlying psychosocial factors and vaccination response associations and the future research needed to understand these better, and indeed to use current and future knowledge to improve and enhance vaccine responses in key at risk populations.

Key words: caregiving; influenza; interventions; social support; stress; vaccine;

\section{Introduction: Why study vaccination in the context of PNI research?}

\subsection{Alternative approaches: enumerative measures}

There are many methods for examining the effects of psychological factors on immunity. Early work concentrated on the influence of psychosocial stress on enumerative measures of immunity. For example, individuals exposed to chronic stress showed reduced numbers of certain immune cells including reduced numbers of B-lymphocytes $(\mathbf{1 , 2})$, helper T-lymphocytes $(\mathbf{3 , 4 , 1 )}$, 
cytotoxic T-lymphocytes $(5,1)$, natural killer $(\mathrm{NK})$ cells $(5,1)$ and lowered concentrations of secretory immunoglobulin A in saliva (6-10), compared to matched controls. However, it is difficult to determine the clinical significance of such enumerative changes, given that they lie within the normal range for healthy participants (11) and may simply reflect cell migration and recirculation rather than increased production or better function (11). Additionally, cell number changes could be a consequence of shifts in plasma volume and haemoconcentration; in such circumstances, changes in cell number would reflect increased density of a lymphocyte population rather than signal a true increase in absolute cell numbers. Further, even absolute changes in cell number might not necessarily reflect alteration in the capacity of the immune system to mount an effective response to antigenic challenge (11). Consequently, measuring changes in cell number is perhaps not the optimal means of determining variations in the functional capacity of the immune system, and hence the likely clinical implications of psychosocial variables for disease resistance and susceptibility.

\subsection{In vitro measures}

In vitro measures of immune function, such as cell proliferation to stimulation with an antigen (foreign material e.g. bacteria), or cell cytotoxicity (killing ability), have been argued to provide a better indication of the functional capacity of the immune system (11). These measures have been demonstrated to be susceptible to impairment by chronic stress in many studies, e.g., (1215). For example, recently unemployed individuals showed poorer lymphocyte proliferation to antigen than those in employment (16). Further, compared to non-bereaved controls, individuals who have suffered bereavement showed lower neutrophil superoxide production, one of their key 
cytotoxic capacities through which they eradicate bacteria such as pneumonia (17).

Nevertheless, the isolated testing of any particular network of immune cells provides only limited information about the overall status of what is a highly integrated and complex system (11), and an imperfect understanding of the relationship between psychosocial factors and vulnerability to disease (18).

\section{The vaccination model}

\subsection{Benefits of the vaccination model}

A clinically relevant model which examines the impact of psychosocial factors on the integrated response of the immune system to a challenge would avoid these disadvantages. The antibody response to vaccination provides us with such a model. Vaccines act as real immune system challenges, although they are altered in such a way so not to induce disease either by being inactivated or killed, or only a component of the actual pathogen, so are really 'imitation infections'. Therefore, by measuring the antibody levels in response to vaccination we can assess directly how well the immune system responds to infectious challenge. It is also clinically relevant in that antibody levels or titres are directly related to susceptibility and resistance to infectious disease.

\subsection{The vaccination response}

The vaccination response involves the coordination of a wide variety of immune cells. Antigen is initially recognized and presented by professional antigen presenting cells, such as dendritic 
cells. Thus presented, the antigen is then recognised by specific helper $\mathrm{T}$ cells which process and present the antigen to B-cells, the antibody factories of the immune system; this is termed a thymus-dependent response. There are other types of vaccination which are also recognized by B-cells without the necessity for T-cell help, thus termed a thymus-independent response, which do not elicit as strong or maintained a response as thymus-dependent vaccines. A final type of vaccination, called a conjugate vaccination, is used to improve the response to thymusindependent antigens by attaching a protein to the antigen, which then stimulates an immune response involving helper T-cell recognition.

When stimulated by an antigen, B-lymphocytes replicate and mature into short lived plasma cells which produce the earliest antibody or immunoglobulin, IgM. In a primary response to an antigen not previously encountered, the peak IgM response occurs around five days after vaccination. Interaction between activated T- and B-cells leads to the production of high affinity or very specific antibodies in bodily fluids: IgG (found mainly in the blood) and IgA (found mainly at mucosal surfaces, e.g. in saliva). This more specific response peaks around 28 days after vaccination. Other types of antibody include $\operatorname{IgE}$ (part of the allergic response) and $\operatorname{IgD}$. $\operatorname{IgG}$ is particularly important, as being the most prolific antigen in the blood and a more specific match to the particular antigen makes it more effective at antigen elimination. Secondary antibody responses, in which the immune system has been previously exposed to the antigen, are more rapid and of greater magnitude; this is because some activated T- and B-cells become long lived resting memory cells, remaining in the immune system ready to respond quickly to challenge with previously encountered antigens. Part of the response to vaccination is also cellmediated as well as humoral (involving antibodies) such that helper T-cells are also activated and 
initiate production of key pro-inflammatory cytokines, including IL-1 $\beta$, IL-6, and IL-8, in response to vaccine antigens, which can also be measured quantitatively as part of the vaccination response, although is less commonly done so.

Not all individuals react with a strong antibody response to vaccination, particularly older adults who are only protected against influenza disease in 30-50\% of cases following vaccination (1922). Further, the increase in vaccination availability has not been paralleled by decreased influenza-related mortality (23). This variation in the vaccination response allows for the investigation of other factors which might influence this aspect of immunity between individuals. The relevance of the response to infectious disease risk provides the clinically relevant imperative to do so beyond the interest in increasing knowledge on how various factors affect immune function. As well as age, psychosocial factors, such as stress, may alter both the quantity and quality of antibody present at different times after immunization, meaning that individuals suffering higher levels of stress are more at risk of infectious disease.

\section{Stress and the vaccination response}

\subsection{Stress questionnaires}

The most common psychosocial factor examined in the context of the vaccination response is stress. This is usually assessed via life events checklists or perceived stress measures. Life events checklists consist of a list of major and minor life events, e.g. bereavement, moving house, and usually require participants to indicate which have occurred during the past month or 
year (24). Some also ask participants to indicate how stressful each event was on a rating scale.

Life events have been shown to predict a variety of important physical health outcomes, including infectious disease (25), and mortality, particularly in the context of little emotional support (26). In contrast, perceived stress scales measure individuals' feelings about how stressful their lives are rather than the direct occurrence of events (27). Thus these measures are more susceptible to subjective bias, and are better predictors of subjective health outcomes, such as angina, rather than objective outcomes, such as myocardial infarction (28).

\subsection{Caregiver control models}

Another common way of assessing stress in the context of vaccination is to examine antibody responses among those with a key chronic stressor versus a socio-demographically matched control group, for example, older adults caregiving for a spouse with dementia. The stress of caregiving has been shown to relate to poor health and mortality (29), and can thus be considered an important source of ongoing psychological stress. Other stress studies compare matched controls to other groups subject to chronic stressors such as bereavement, marital separation/divorce, or unemployment.

\subsection{Protocol for stress and vaccination studies}

In order to fully test the impact of psychosocial factors on the response to vaccination, both prevaccination and post-vaccination blood samples are required for assessment of antibody levels. This is due to the impact that prior vaccination or environmental exposure to the infectious agent 
can have on pre-vaccination antibody levels, and consequently post-vaccination levels. Without taking a pre-vaccination baseline, it is difficult to state whether stress is affecting the antibody response to a vaccination administered during a research study or simply on the maintenance of previous antibody levels. For example, in 37 nursing home residents, those who reported higher levels of perceived stress had lower pre-vaccine antibody titres to two influenza vaccine components (30). However, it is not clear what this means, given that pre-vaccine titres could reflect differences in prior vaccine history or exposure. In this same study, social support was also negatively correlated with pre- and post-vaccination titres against the A/Panama influenza strain yet positively with pre-vaccination antibody titres against the A/New Caledonia strain (30), making interpretation of the findings very difficult. However, some of the early studies of stress and vaccination in students were opportunistic, in other words they collected stress data from students who opportunistically had already received a prior vaccination. Although more complex to interpret, given lack of baseline or prior exposure information, these studies are able to show that psychological stress does seem to affect the maintenance of antibody titres over time. For example, one study examined the association between life events stress and hepatitis B antibody titre in medical students, vaccinated either in the past twelve months or at least thirteen months previously (31). Whereas life events exposure was not related to antibody response in the recently vaccinated cohort, participants in the earlier vaccinated cohort who reported higher life events over the past year were over twice as likely to show an inadequate antibody titre as those with lower life events exposure, providing some evidence that psychosocial stress can have effects on the rate of deterioration of antibody protection (31). Similarly, the maintenance of higher antibody levels after the conjugate meningococcal $\mathrm{C}$ vaccination was associated with lower perceived stress (32). 


\subsection{Key stress and vaccination findings}

One of the most common vaccinations studied in the context of stress and antibody response is the influenza vaccination, particularly in undergraduate student and older caregiver samples. The influenza vaccination is a commonly utilised vaccine and consists of three components or strains, usually two A strains and one B strain, which change each year depending on the key circulating varieties. A meta-analysis of 13 studies of psychological stress and influenza vaccination concluded that there is a significant negative relationship between psychological stress and antibody titre following influenza vaccination (33). These studies included five in caregivers and eight assessing the impact of stressful life events or perceived stress. The metaanalysis concluded that psychological stress, however measured, had a similar negative impact on influenza vaccine response, but that antibody responses to A/H1N1 and B-influenza types were more sensitive to the influence of stress (33). However, it is difficult at this stage to explain why antibodies against influenza strains are differentially associated with stress. One possibility is that strain novelty influences the associations observed $(\mathbf{3 4 , 1 1})$, with more novel strains being more susceptible to stress effects.

The impact on certain A-strains and on B-strains is clearly shown in several studies of students. For example, those reporting higher stressful life event exposures and/or higher perceived stress prior to vaccination showed poorer responses to the A-strains of the vaccine at five weeks (around the time of the peak response) and five months post-vaccination (indicating the decay in antibody response over time) (35). This was replicated for the numbers and severity of stressful 
life events prior to vaccination with the response to the B/Shandong influenza strain at both five weeks and five months post-vaccination (36). Similarly, in a study of the effects of daily stress and feelings of being overwhelmed during the 10 days following vaccination, higher stress ratings were associated with lower antibody titres to the A/New Caledonia strain at both one and four months following vaccination (37). In older adults too, we observed that the stress of bereavement in the year prior to influenza vaccination was associated with a poorer antibody response to two of the influenza strains in a community sample of 184 adults aged 65 and over (38). Overall negative life events exposure was not associated with vaccine response in this study, as has similarly been found for self-reported stress in another study of the influenza vaccination in older adults, although only one item was used to assess stress (39). However, the effect found above for bereavement suggests that stressful events are related to pervasive immune effects throughout the life course, although what constitutes life events stress will vary depending on the age of the sample studied. Taken together, these studies provide evidence that stressful life events both preceding and in the period immediately following vaccination can influence the antibody response. They also show that both the peak antibody response at around four weeks and the decay in antibody protection over time are susceptible to influence by stressful life events.

Interestingly, in the majority of these studies, where measured, self-reported or perceived stress has not been associated with the antibody response to vaccination, whereas the occurrence of stressful life events has. This suggests that actual stressful events occurrence is more detrimental than individuals' perceptions of stress, which may more closely reflect individual differences in personality and coping style than a quantitative count of stressful occurrences. 
On the whole, the vaccination response in older adults has mainly been considered in the context of the chronic stress of caregiving for a spouse with dementia. Studies have shown that caregivers have poorer antibody responses to vaccination in comparison to matched control participants (40-42). Similarly, caregivers who exhibited repetitive negative thoughts about their situation had lower antibody titres following influenza vaccination (43). More recently, in a study in Hong Kong, older caregivers had significantly lower cell-mediated but not humoral (antibody) responses to the influenza vaccination at 12 weeks compared to non-caregivers (44). However, in younger populations, such as Multiple Sclerosis spousal caregivers, there was no difference in antibody response to influenza vaccination between caregivers and controls (45). This raises the issue of whether the poor antibody response observed in older caregivers is, to an extent, a function of an interaction between chronic stress exposure and immunosenescence (46).

There is an alternative explanation for the discrepancy in outcomes among the caregiver vaccination studies. Rather than immune ageing, perhaps it is the intensity of the stress experienced that determines whether caregiving becomes an issue for immunity (45). Dementia is a disease characterised by much more severe cognitive and behavioural disturbances than multiple sclerosis (47-50), and older spousal caregivers of dementia patients have been found to report greater distress than younger multiple sclerosis caregivers (45). Further, the results of two recent meta-analyses indicate that caregivers of dementia patients generally experience greater burden and report more symptoms of depression than those caring for non-dementia, e.g., cancer, patients, $(\mathbf{5 1 , 5 2 )}$. Thus, it might be 
hypothesized that, irrespective of the caregiver's age, caring for someone with severe cognitive and behavioural problems will compromise immunity.

We have been able to test this hypothesis recently using a caregiving model in younger adults; young parents caring for children with developmental disabilities. Dealing with severe cognitive difficulties and behaviours that are problematic and distressing are the main challenges of such caring (53-56). In our own studies of 30 caregivers for a child with a developmental disability (mainly Autism) versus matched controls, we have demonstrated that caregivers report high levels of stress, anxiety, depression, child problem behaviours, and low levels of social support. These caregivers also exhibited a poorer antibody response to a pneumonia vaccination than parents caring for typically developing children at both one and six months post-vaccination (57). Of the psychological variables considered, child problem behaviours mediated this effect. In addition, within the caregivers, parents reporting more child conduct problems, a component of the child problem behaviour measure, mounted a poorer antibody response at 1-month than parents reporting less conduct problems (57). Similarly, these parents mounted a poorer antibody response to the B/Malaysia strain of an influenza vaccine at one and six months post-vaccination, which again appeared to be mediated by differences in child problem behaviours (58).

These recent findings in younger caregivers reinforce the hypothesis that an ageing immune system is not a pre-requisite for a poor response to medical vaccination in caregivers. Nevertheless, among our parental caregivers, older caregivers tended to have a poorer 
antibody response to B/Malaysia at 1-month, suggesting that we cannot dismiss the hypothesis that chronic stress and immunosenescence may have synergistic effects (46).

\section{Different vaccine factors}

Vaccination studies also have the advantage of being able to incorporate research questions such as 'does it matter when stressful life events occur relative to when the vaccine is received?' and 'what are the effects of prior exposure to the antigen?' The next section of this chapter will address some of these issues of timing.

\subsection{Timing of stress measurement}

This issue of the timing of stress assessment has been developed in studies of various vaccinations including hepatitis $\mathrm{B}$, which is useful in this context, as the vaccination schedule consists of three inoculations over a six month period. The largest of these studies examined the association between life events stress and the final antibody titre in students, vaccinated either in the past twelve months or at least thirteen months previously (31). Whereas life events exposure was not related to antibody response in the recently vaccinated cohort, participants in the earlier vaccinated cohort who reported higher life events over the past year were over twice as likely to show an inadequate antibody titre as those with lower life events exposure. This finding suggests that the immunogenicity, the ability to induce a strong vaccination response, of hepatitis B vaccination may initially override the influence of life events stress, although there was also more power to detect effects in the earlier vaccinated cohort as more participants exhibited 
inadequate antibody titres (31). Nevertheless, this study provides some evidence that psychosocial stress in the period following vaccination can have effects on the rate of deterioration of antibody protection (18).

In a study where a low dose of hepatitis B vaccine was administered, a higher stress index, comprising life events exposure and psychological symptoms, measured at two months postvaccination (thus considering the period post-vaccination) was associated with a poorer final six month antibody response, and the stress index at six months also tended to relate negatively to antibody response (59). However, as only the final antibody titre was measured, it is difficult to determine whether, in this instance, stress predominantly influenced initial formation or maintenance of antibody levels. Also, the inclusion of psychological symptoms in the composite stress index makes it difficult to ascribe this finding to any specific aspect of stress (18). A similar study using the full dosage hepatitis B vaccination did not yield any significant stress effects (60), although it is possible that this was due to the absence of a two-month assessment of stress, which was the main predictor of antibody response in the previous study by this group. In a study measuring perceived stress and anxiety during the vaccination period, i.e. postvaccination, these were not associated with the final antibody response to hepatitis B (40). Further, life events stress prior to vaccination and perceived stress at the time of the initial vaccination were not related to antibody status five months following the initial inoculation in a more recent study (61). On the whole, this would suggest either that stress prior to vaccination is less detrimental to the antibody response than stress post-vaccination, or that it is difficult to observe stress effects early on with the full dose hepatitis B vaccination, due to its 
immunogenicity. Given the findings with the influenza vaccination and stress, this latter seems the more likely explanation.

In contrast to the studies of hepatitis B discussed thus far, one study reported a positive association between perceived life event stress, depression and anxiety during the vaccination period and hepatitis B antibody status nine months following the initial vaccination (62). This anomalous result has been attributed to the relatively low levels of stress experienced by the participants in this study, suggesting that moderate levels of life change stress experienced during the initial stages of antibody formation may be beneficial to the antibody response, although high levels may be detrimental (62). Such an interpretation receives support from animal research where moderate stress at the time of vaccination has been associated with an enhanced antibody response (see e.g. (63). This will be discussed further in the section on acute stress below.

\subsection{Primary and secondary exposure to vaccine antigens}

Vaccination with an antigen to which the participant has not been previously exposed induces a primary antibody response whereas vaccination against more common pathogens such as influenza, induce a secondary immune response. By examining the effect of stress on both primary and secondary immune responses, we can begin to determine which aspects of the immune response are most susceptible to stress-induced modulation. 
Hepatitis B vaccination has been used in this context due to the vaccination schedule and the low likelihood of prior naturalistic exposure to this pathogen. In an earlier study, individuals reporting higher mean perceived stress and anxiety over the vaccination period were less likely to have sero-converted (produced a protective antibody level) by the time of the second inoculation (40). Whereas, an emotional disclosure intervention group did not differ from controls in antibody levels at the time of the second inoculation (64). However, psychological stress levels were not measured, making it difficult to interpret these data. More recently, we have used hepatitis A as a primary antigen. Students who reported a higher number and severity of life events had a poorer antibody response to hepatitis A at the 18-week, but not 4-week, follow-up, suggesting stress can impact upon the maintenance of antibody levels (65). Early studies using the vaccination model used novel non-pathogenic antigens to examine the antigenspecific antibody response. Keyhole limpet hemocyanin (KLH), a protein, has been used in this context; the KLH-specific IgG antibody response was lower at eight weeks, but not three weeks, post-vaccination in participants reporting fewer positive life events prior to vaccination (66).

The consensus of this evidence suggests that stress can influence the primary antibody response, particularly the maintenance of responses to novel antigens. It also supports the idea that life events stress effects are more likely to be evident with novel vaccine types (36). As discussed above, the secondary antibody response to hepatitis B vaccination has produced mixed results, but there appears to be stronger evidence for a negative effect of psychological stress on the secondary response to this antigen $(18,67)$, in line with the findings for the influenza vaccine. 


\subsection{Thymus-dependent versus thymus-independent vaccines}

A further advantage to the vaccination model is that there are different types of vaccination, which can be used to help elucidate which cells involved in the vaccination response are influenced by psychological factors. Most vaccinations, which consist of inactivated or dead viruses like influenza, induce a thymus-dependent antibody response, as described above. A few vaccinations, however, protect against bacterial infections or toxins, like meningococcal A or tetanus, respectively, which do not require T-cell help. There are also conjugate vaccines, in which substances that elicit a T-cell response are conjugated to a thymus-independent pathogen, such as a protein, in order to boost the efficiency of the antibody response against the thymusindependent pathogen. If psychological factors are consistently associated with the response to thymus-dependent and conjugate vaccinations but not with thymus-independent response, this would imply that it is T-cells that are particularly liable to psychological influence.

Indeed, there is evidence to suggest that stress may exert its effects mainly on T-cells; we showed that higher frequency and intensity of stressful life events were associated with a poorer response to influenza and meningococcal $\mathrm{C}$ (following previous conjugate meningococcal $\mathrm{C}$ vaccination), but not to thymus-independent meningococcal A (36). Similarly, no association was found between stress and antibody response to a thymus-independent pneumonia vaccination in pre-school children (68). However, as older care-givers have been reported to show poorer maintenance of antibody levels over time following pneumonia vaccination than controls (41), it is possible that other factors such as age and severity of stress may interact to impair antibody-mediated immunity more generally than just the T-cell response. 
It should be noted that in the study of caregivers and the pneumonia vaccination, perceived stress did not differ between the caregiver and controls, but there was a significant difference in social support. This might suggest that thymus-dependent vaccinations are susceptible to the effects of stressful life events, but that thymus-independent vaccinations are more vulnerable to other psychosocial factors such as lower social support. There is some evidence for this suggestion. In our own laboratory, we found that social support, but not life events stress, was positively associated with the response to a thymus-independent pneumococcal vaccine in young healthy students $(65,69)$.

The comparison between thymus-dependent and -independent vaccination responses suggest that both types of response are susceptible to psychosocial influence, but that there are key variables which influence whether an effect on vaccination response is observed. These include: the type of psychosocial factor studied (i.e. stress versus social support), and the age of the population sampled.

\subsection{Acute versus chronic stress}

Following on from the discussion above in section 4.1 regarding when stress is measured, such that moderate or less severe stress at the time of vaccination might actually have a beneficial effect, it has been suggested in recent years that acute (minutes or hours) stress may be immune enhancing when experienced close to the immune challenge. Such immune enhancement by 
acute stress would be an adaptive mechanism, and might be regarded as an integral component of the fight or flight response, and circumstances that elicit such a response are likely to also involve exposure to antigens and, therefore, a robust immune response would be adaptive for survival (63). Our laboratory examined the effect of acute psychological stress on antibody response to vaccination in humans. Participants completed a 45 min time pressured, socially evaluated mental arithmetic task, or a resting control period, immediately prior to influenza vaccination. An enhancement of the antibody response to one of the influenza viral strains was found in women in the psychological stress group compared to control (70). Similarly, in men, the antibody response to a meningococcal A vaccination was enhanced by acute psychological stress (71). That these effects emerged for only one gender or the other in these studies might be explained by examining the antibody responses for each gender. In each case, stress was associated with the antibody response in those with the poorest increase in antibodies in response to vaccination; women for the influenza A/Panama strain and men for the meningococcal A vaccine. This latter study (71) also provides further evidence that both thymus-dependent and independent vaccinations are responsive to the impact of stress, as discussed above. Further, although not a psychological stressor per se, acute eccentric exercise (arm contractions) was also shown to enhance the antibody response to influenza vaccination in women, and the cellmediated IFN $\gamma$ production response to stimulation with the influenza vaccine in men (72). This and similar studies have also lent weight to the contention that effects of behavioural factors on vaccination responses are most likely to be observed in groups with the poorest antibody response, or to vaccine strains which are not very immunogenic i.e. they engender lower

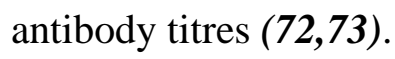




\subsection{Timing of vaccination}

As well as the timing and duration of stress measurements associated with the vaccine response, other behavioural factors have been found to impact upon the antibody response. One such factor is that of the time of day of vaccination. In our study of the effects of psychological stress on vaccination responses in the 184 older adults $(38)$, we observed that the time of day of vaccine administration significantly influenced antibody titre (74). Men responded better in the morning than the afternoon; $41 \%$ of men showed a two-fold response when vaccinated in the morning versus $24 \%$ of men vaccinated in the afternoon. This effect was independent of current illnesses, medication, vaccination history, and our reported findings of the effects of bereavement and marital quality. Women tended to show the reverse pattern. We also observed the same pattern in a study of younger adults' antibody response to the hepatitis A vaccination (74). However, these studies were not fully randomised, and there was little opportunity to examine the biological mechanisms, such as cytokine and stress hormone levels. Consequently, there was a clear and pressing need for a randomised controlled trial of the impact of time of influenza vaccination on antibody response and vaccine efficacy in older adults in an National Health Service (NHS) setting. We were able to conduct such a cluster-randomised trial within the National Health Service in Birmingham, UK, and confirmed that a simple manipulation of the time of vaccination can improve the immune response against influenza in older adults. Two hundred and seventy-six participants were randomized to have the annual influenza vaccination at one of 24 General Practice surgeries in either the morning (9-11am) or afternoon (3-5pm). This trial provided some evidence that morning vaccination produced higher antibody levels, at least for the H1N1 A-strain with a trend for the same effect for the B influenza strain $(\mathbf{7 5 , 7 6 ) .}$ Consequently, although this requires a larger scale trial for confirmation, we believe that 
morning vaccination could be employed as an easy to adopt intervention within the health services, at little or no added cost. The potential benefits would be a decreased incidence of influenza infection and influenza-related mortality in older adults; although a multi-center very large trial following up on verified influenza incidence and health outcomes would be essential to fully prove this.

\section{Other psychosocial factors and the vaccination model}

\subsection{Social support}

The support of friends and loved ones is an important determinant of immune health, and is relatively easily measured in vaccination studies via validated questionnaires. Studies have assessed both functional social support, a measure of the quality and availability of social resources a person has, and structural social support, the number of friends a person can call on, in the context of vaccination. First, students reporting greater social support demonstrated a stronger combined immune response to the third inoculation of the three-dose hepatitis B vaccination (40). Second, loneliness and smaller social network size were associated with a poorer antibody response to the A/New Caledonian strain of the influenza vaccination in college students (77). Third, students with greater functional social support showed higher titres to the A/Panama influenza strain at both five weeks and five months following vaccination (36). In older nursing home residents, social support was also negatively correlated with pre- and postvaccination titres against the A/Panama influenza strain yet positively with pre-vaccination antibody titres against the $\mathrm{A} / \mathrm{New}$ Caledonia strain (30), a finding which even the authors were unable to explain. Along with the caregiver study discussed above, these studies generally show 
that a lack of social support has a strong negative impact on antibody levels following vaccination.

Marriage is also a source of social support. In our own work, older adults who were married, and particularly those who were happily married, showed a better antibody response to the influenza vaccination than those who were unmarried or less happily married (38). However, more general functional social support and social network size was not associated with antibody response in this older population (38). Further, for children, the relationship with their parents is the key source of support, and negative parent-child interactions such as conflict have been associated with a less robust antibody response to the meningococcal $\mathrm{C}$ vaccination over six months (78). These findings perhaps lend weight to the suggestion that the age of the population studied influences which psychosocial factors are important for the vaccination response.

\subsection{Personality}

Personality factors, although often examined in the context of health outcomes, again using validated questionnaires (see e.g., (79), have scarcely been investigated relative to the vaccination response. First, among a group of 12-year old girls, those characterized by higher internalizing scores and lower self-esteem at baseline exhibited lower antibody titres following rubella vaccination (80). A similar concept, neuroticism, was negatively associated with both the peak antibody response to the A/Panama strain of an influenza vaccination, and the maintenance of antibody titres to this strain in students (81). Among female graduate students, trait negative affect/mood was negatively associated with the antibody response to the second hepatitis B injection (61). Further, independently of negative affect, trait positive affect was associated with 
a better antibody response following a second hepatitis B vaccination in graduate students (82). Thus, both negative and positive traits appear to be able to influence this aspect of immune function and disease protection. However, in exercising and sedentary elderly individuals, dispositional optimism was not found to be associated with antibody titres following influenza vaccination (83). Inconsistencies in these results could be attributable to the different measures of personality studied, or the different ages of the populations used, which will now be discussed in more detail.

\section{Future directions: mechanisms and interventions}

The studies reviewed above outline the different methods of examining associations between psychological factors and the antibody response to vaccination. These studies show the strong associations between psychological stress, other psychosocial factors and the immune response to vaccination, such that stressful psychological circumstances are associated with poorer antibody responses, while positive factors such as social support relate to a better immune response to vaccination. Taken together, these findings suggest two main directions for future

research. First, despite the range of vaccinations used in such studies, as yet little is known about the exact mechanisms by which stress and other factors can influence antibody responses to vaccination. Research incorporating a range of measurements, such as stress hormones, immune system messengers (cytokines), and the function of key cells in the vaccination response, such as antigen presenting cells, would be necessary to further our understanding regarding exactly how stress gets inside the body to affect this clinically relevant immune outcome. Second, the clinical implications, in terms of susceptibility to disease, arising from a better understanding of the 
relationships between psychological factors and the vaccination response are important, particularly in the context of older adults who already display poor vaccination responses. Psychological interventions to improve vaccination response in these populations could include techniques such as stress management, relaxation, cognitive behavioural therapy, and emotional disclosure.

Regarding such interventions, one study showed an improvement in the ability of older caregivers for a spouse with dementia to mount a four-fold increase in antibody titre following influenza vaccination relative to matched controls, although the mechanisms of effect were unclear and the intervention group was not randomly sampled (84). Similarly, participants taking part in a written emotional disclosure intervention, where they wrote about their emotions about a previously undisclosed stressful event, showed significantly higher antibody titres at four and six months following vaccination with hepatitis B compared to a control non-intervention group (64). A different clinical application of the vaccination model has arisen from the positive immune effects demonstrated in response to acute stress and exercise, as discussed above (70). These preliminary findings suggest that the development of such a behavioural challenge that could be applied in General Practitioner settings could be a way forward for improving the vaccination response. This would be particularly important for groups at risk of infectious disease such as older adults, the bereaved, and care-givers. At this stage, more work is required to establish exactly what types of intervention in which age groups and are likely to be the most beneficial for psychological, and hence immunological, health. Behavioural interventions, such as the time of day of vaccination may also be important in this context. 


\section{Conclusion}

In conclusion, vaccination has had a substantial impact on public health, although not everyone mounts a satisfactory and protective antibody response to vaccination. This increasingly appears to be the case with progressing age. Studying antibody responses to vaccination is now contributing to the understanding of how psychosocial exposures can influence immunity and, consequently, resistance to disease. The current challenges are to build upon the methodology that has been developed through these studies to unravel the underlying mechanisms and to develop and apply feasible behavioural interventions to boost the response to vaccination and, thus, optimize our resistance against infectious disease. 


\section{References}

1. McKinnon W, Weisse CS, Reynolds CP et al. (1989) Chronic stress, leukocyte

subpopulations, and humoral response to latent viruses. Health Psychol 8:389-402

2. Schaeffer MA, Baum A, Reynolds CF et al. (1985) Immune status as a function of chronic stress at Three Mile Island. Psychosom Med 47:85

3. Futterman AD, Wellisch DK, Zighelboim J et al. (1996) Psychological and immunological reactions of family members to patients undergoing bone marrow transplantation. Psychosom Med 58:472-480

4. Kiecolt-Glaser JK, Glaser R, Shuttleworth EC et al. (1987) Chronic stress and immunity in family caregivers of Alzheimer's disease victims. Psychosom Med 49:523-535

5. De Gucht V, Fischler B, Demanet C (1999) Immune dysfunction associated with chronic professional stress in nurses. Psychiatry Res 85:105-111

6. Deinzer R, Kleineidam C, Stiller-Winkler R et al. (2000) Prolonged reduction of salivary immunoglobulin A (sIgA) after a major academic exam. Int J Psychophysiol 37:219-232 7. Jemmott JB, 3rd, Borysenko JZ, Borysenko M et al. (1983) Academic stress, power motivation, and decrease in secretion rate of salivary secretory immunoglobulin A. Lancet 1:1400-1402

8. Jemmott JB, 3rd, Magloire K (1988) Academic stress, social support, and secretory immunoglobulin A. J Pers Soc Psychol 55:803-810

9. McClelland DC, Alexander C, Marks E (1982) The need for power, stress, immune function, and illness among male prisoners. J Abnorm Psychol 91:61-70

10. Gallagher S, Phillips AC, Evans P et al. (2008) Caregiving is associated with low secretion rates of immunoglobulin A in saliva. Brain Behav Immun 22:565-572. doi:S0889-

1591(07)00309-1 [pii]10.1016/j.bbi.2007.11.007

11. Vedhara K, Fox JD, Wang ECY (1999) The measurement of stress-related immune dysfunction in psychoneuroimmunology. Neuroscience and Biobehavioural Reviews 23:699-715 12. Bartrop RW, Luckhurst E, Lazarus L et al. (1977) Depressed lymphocyte function after bereavement. Lancet 1:834-836

13. Esterling BA, Kiecolt-Glaser JK, Glaser R (1996) Psychosocial modulation of cytokineinduced natural killer cell activity in older adults. Psychosom Med 58:264-272 
14. Irwin M, Patterson T, Smith TL et al. (1990) Reduction of immune function in life stress and depression. Biol Psychiatry 27:22-30

15. Schleifer SJ, Keller SE, Camerino M et al. (1983) Suppression of lymphocyte stimulation following bereavement. JAMA 250:374-377

16. Arnetz BB, Wasserman J, Petrini B et al. (1987) Immune function in unemployed women. Psychosom Med 49:3-12

17. Khanfer R, Lord JM, Phillips AC (2011) Neutrophil function and cortisol:DHEAS ratio in bereaved older adults. Brain Behav Immun. doi:S0889-1591(11)00076-6

[pii]10.1016/j.bbi.2011.03.008

18. Burns VE, Carroll D, Ring C et al. (2003) Antibody response to vaccination and psychosocial stress in humans: relationship and mechanisms. Vaccine 21:2523-2534

19. Allsup S, Haycox A, Regan M et al. (2004) Is influenza vaccination cost effective for healthy people between ages 65 and 74 years? A randomised controlled trial. Vaccine 23:639-645 20. Patriarca PA (1994) Editorial: A randomized controlled trial of influenza vaccine in the elderly: Scientific scrutiny and ethical responsibility. JAMA 272:1700-1701

21. Goodwin K, Viboud C, Simonsen L (2006) Antibody response to influenza vaccination in the elderly: a quantitative review. Vaccine 24:1159-1169. doi:S0264-410X(05)00955-2 [pii]10.1016/j.vaccine.2005.08.105

22. Nichol KL, Margolis KL, Wuorenma J et al. (1994) The efficacy and cost effectiveness of vaccination against influenza among elderly persons living in the community. New Engl J Med 331:778-784

23. Simonsen L, Reichert TA, Viboud C et al. (2005) Impact of influenza vaccination on seasonal mortality in the US elderly population. Arch Intern Med 165:265-272

24. Brown GW, Harris TO (1989) Social Origins of Depression: A study of psychiatric disorder in women. Routledge, London

25. Cohen S, Tyrell DAJ, Smith AP (1991) Psychological stress and susceptibility to the common cold. New Engl J Med 325:606-612

26. Rosengren A, Orth-Gomer K, Wedel H et al. (1993) Stressful life events, social support, and mortality in men born in 1933. BMJ 307:1102-1105

27. Cohen S, Kamarck T, Mermelstein R (1983) A global measure of perceived stress. J Health Soc Behav 24:385-396. doi:http://dx.doi.org/10.2307/2136404 
28. Macleod J, Davey Smith G, Heslop P et al. (2002) Psychological stress and cardiovascular disease: empirical demonstration of bias in a prospective observational study of Scottish men. BMJ 324:1247-1251

29. Schulz R, Beach SR (1999) Caregiving as a risk factor for mortality: the Caregiver Health Effects Study. JAMA 282:2215-2219

30. Moynihan JA, Larson MR, Treanor J et al. (2004) Psychosocial factors and the response to influenza vaccination in older adults. Psychosom Med 66:950-953

31. Burns VE, Carroll D, Ring C et al. (2002) Stress, coping and hepatitis B antibody status. Psychosom Med 64:287-293

32. Burns VE, Drayson M, Ring C et al. (2002) Perceived stress and psychological well-being are associated with antibody status following meningitis $\mathrm{C}$ conjugate vaccination. Psychosom Med 64:963-970

33. Pedersen AF, Zachariae R, Bovbjerg DH (2009) Psychological stress and antibody response to influenza vaccination: a meta-analysis. Brain Behav Immun 23:427-433. doi:S0889-

1591(09)00007-5 [pii]10.1016/j.bbi.2009.01.004

34. Gulati U, Keitel WA, Air GM (2007) Increased antibodies against unfolded viral antigens in the elderly after influenza vaccination. Influenza Other Respi Viruses 1:147-156. doi:10.1111/j.1750-2659.2007.00017.x

35. Burns VE, Carroll D, Drayson M et al. (2003) Life events, perceived stress and antibody response to influenza vaccination in young healthy adults. J Psychosom Res 55:569-572 36. Phillips AC, Burns VE, Carroll D et al. (2005) The association between life events, social support and antibody status following thymus-dependent and thymus-independent vaccinations in healthy young adults. Brain Behav Immun 19:325-333

37. Miller GE, Cohen S, Pressman S et al. (2004) Psychological stress and antibody response to influenza vaccination: when is the critical period for stress, and how does it get inside the body? Psychosom Med 66:215-223

38. Phillips AC, Carroll D, Bums VE et al. (2006) Bereavement and marriage are associated with antibody response to influenza vaccination in the elderly. Brain Behav Immun 20:279-289. doi:10.1016/j.bbi.2005.08.003 
39. Talbot HK, Coleman LA, Zhu Y et al. (2015) Factors associated with maintenance of antibody responses to influenza vaccine in older, community-dwelling adults. BMC Infect Dis 15:195. doi:10.1186/s12879-015-0926-8

40. Glaser R, Kiecolt-Glaser JK, Bonneau RH et al. (1992) Stress-induced modulation of the immune response to recombinant hepatitis B vaccine. Psychosom Med 54:22-29

41. Glaser R, Sheridan JF, Malarkey W et al. (2000) Chronic stress modulates the immune response to a pneumococcal pneumonia vaccine. Psychosom Med 62:804-807

42. Vedhara K, Cox NK, Wilcock GK et al. (1999) Chronic stress in elderly carers of dementia patients and antibody response to influenza vaccination. Lancet 353:627-631

43. Segerstrom SC, Schipper LJ, Greenberg RN (2008) Caregiving, repetitive thought, and immune response to vaccination in older adults. Brain Behav Immun 22:744-752. doi:S08891591(07)00307-8 [pii]10.1016/j.bbi.2007.11.004

44. Wong SY, Wong CK, Chan FW et al. (2013) Chronic psychosocial stress: does it modulate immunity to the influenza vaccine in Hong Kong Chinese elderly caregivers? Age (Dordr) 35:1479-1493. doi:10.1007/s11357-012-9449-z

45. Vedhara K, McDermott MP, Evans TG et al. (2002) Chronic stress in non-elderly caregivers: psychological, endocrine and immune implications. J Psychosom Res 53:1153-1161

46. Graham JE, Christian LM, Kiecolt-Glaser JK (2006) Stress, age, and immune function: toward a lifespan approach. J Behav Med 29:389-400

47. Keegan BM, Noseworthy JH (2002) Multiple sclerosis. Annu Rev Med 53:285-302. doi:10.1146/annurev.med.53.082901.10390953/1/285 [pii]

48. Poser CM, Paty DW, Scheinberg L et al. (1983) New diagnostic criteria for multiple sclerosis: guidelines for research protocols. Ann Neurol 13:227-231. doi:10.1002/ana.410130302 49. Gregory CA, Hodges JR (1996) Clinical features of frontal lobe dementia in comparison to Alzheimer's disease. J Neural Transm Suppl 47:103-123

50. Neary D, Snowden JS, Gustafson L et al. (1998) Frontotemporal lobar degeneration: a consensus on clinical diagnostic criteria. Neurology 51:1546-1554

51. Pinquart M, Sorensen S (2003) Associations of stressors and uplifts of caregiving with caregiver burden and depressive mood: a meta-analysis. J Gerontol B Psychol Sci Soc Sci 58:P112-128 
52. Pinquart M, Sorensen S (2003) Differences between caregivers and noncaregivers in psychological health and physical health: a meta-analysis. Psychol Aging 18:250-267. doi:http://dx.doi.org/10.1037/0882-7974.18.2.250

53. Floyd FJ, Gallagher EM (1997) Parental stress, care demands, and use of support services for school-age children with disabilities and behavior problems. Family Relations 46:359-371

54. Hastings RP, Daley D, Burns C et al. (2006) Maternal distress and expressed emotion: crosssectional and longitudinal relationships with behavior problems of children with intellectual disabilities. Am J Mental Retard 111:48-61

55. Higgins DJ, Bailey SR, Pearce JC (2005) Factors associated with functioning style and coping strategies of families with a child with an autism spectrum disorder. Autism 9:125-137 56. Maes B, Broekman TG, Dosen A et al. (2003) Caregiving burden of families looking after persons with intellectual disability and behavioural or psychiatric problems. J Intellect Disabil Res 47:447-455. doi:513 [pii]

57. Gallagher S, Phillips AC, Drayson MT et al. (2009) Parental caregivers of children with developmental disabilities mount a poor antibody response to pneumococcal vaccination. Brain Behav Immun 23:338-346. doi:10.1016/j.bbi.2008.05.006

58. Gallagher S, Phillips AC, Drayson MT et al. (2009) Caregiving for children with developmental disabilities is associated with a poor antibody response to influenza vaccination. Psychosom Med 71:341-344. doi:10.1097/PSY.0b013e31819d1910

59. Jabaaij L, Grosheide PM, Heijtink RA et al. (1993) Influence of perceived psychological stress and distress on antibody response to low dose rDNA hepatitis B vaccine. J Psychosom Res 37:361-369

60. Jabaaij L, Van Hattum J, Vingerhoets AJJM et al. (1996) Modulation of immune response to rDNA hepatitis B vaccination by psychological stress. J Psychosom Res 41:129-137

61. Marsland AL, Cohen S, Rabin BS et al. (2001) Associations between stress, trait negative affect, acute immune reactivity, and antibody response to hepatitis B injection in healthy young adults. Health Psychol 20:4-11

62. Petry J, Weems LB, Livingstone JNI (1991) Relationship of stress, distress, and the immunologic response to a recombinant hepatitis B vaccine. J Family Pract 32:481-486 63. Dhabhar FS, McEwen BS (1996) Stress-induced enhancement of antigen-specific cellmediated immunity. J Immunol 156:2608-2615 
64. Petrie KJ, Booth RJ, Pennebaker JW et al. (1995) Disclosure of trauma and immune response to a hepatitis B vaccination program. J Consult Clin Psychol 63:787-792

65. Gallagher S, Phillips AC, Ferraro AJ et al. (2008) Psychosocial factors are associated with the antibody response to both thymus-dependent and thymus-independent vaccines. Brain Behav Immun 22:456-460

66. Snyder BK, Roghmann KJ, Sigal LH (1990) Effect of stress and other biopsychosocial factors on primary antibody response. J Adolesc Health Care 11:472-479

67. Cohen S, Miller GE, Rabin BS (2001) Psychological Stress and Antibody Response to Immunization: A Critical Review of the Human Literature. Psychosom Med 63:7-18

68. Boyce WT, Adams S, Tschann JM et al. (1995) Adrenocortical and behavioral predictors of immune response to starting school. Pediatric Res 38:1009-1017

69. Gallagher S, Phillips AC, Ferraro AJ et al. (2008) Social support is positively associated with the immunoglobulin $\mathrm{M}$ response to vaccination with pneumococcal polysaccharides. Biol Psychol 78:211-215. doi:S0301-0511(08)00006-9 [pii]10.1016/j.biopsycho.2008.01.001 70. Edwards KM, Burns VE, Reynolds T et al. (2006) Acute stress exposure prior to influenza vaccination enhances antibody response in women. Brain Behav Immun 20:159-168 71. Edwards KM, Burns VE, Adkins AE et al. (2008) Meningococcal A vaccination response is enhanced by acute stress in men. Psychosom Med 70:147-151

72. Edwards KM, Burns VE, Allen LM et al. (2007) Eccentric exercise as an adjuvant to influenza vaccination in humans. Brain Behav Immun 21:209-217

73. Edwards KM, Campbell JP, Ring C et al. (2010) Exercise intensity does not influence the efficacy of eccentric exercise as a behavioural adjuvant to vaccination. Brain Behav Immun 24:623-630. doi:S0889-1591(10)00010-3 [pii]10.1016/j.bbi.2010.01.009

74. Phillips AC, Gallagher S, Carroll D et al. (2008) Morning vaccine administration is associated with an enhanced response to vaccination in men. Psychophysiology 45:663-666 75. Long JE, Drayson MT, Taylor AE et al. (2016) Morning vaccination enhances antibody response over afternoon vaccination: A cluster-randomised trial. Vaccine 34:2679-2685. doi:10.1016/j.vaccine.2016.04.032

76. Long JE, Drayson MT, Taylor AE et al. (2016) Corrigendum to 'Morning vaccination enhances antibody response over afternoon vaccination: A cluster-randomised trial' [Vaccine 34 (2016) 2679-2685]. Vaccine 34:4842. doi:10.1016/j.vaccine.2016.08.031 
77. Pressman SD, Cohen S, Miller GE et al. (2005) Loneliness, social network size, and immune response to influenza vaccination in college freshmen. Health Psychol 24:297-306

78. O'Connor TG, Wang H, Moynihan JA et al. (2015) Observed parent-child relationship quality predicts antibody response to vaccination in children. Brain Behav Immun 48:265-273. doi:10.1016/j.bbi.2015.04.002

79. Smith TW, Glaser K, Ruiz JM et al. (2004) Hostility, anger, aggressiveness, and coronary heart disease: an interpersonal perspective on personality, emotion, and health. J Pers 72:12171270

80. Morag M, Morag A, Reichenberg MA et al. (1999) Psychological variables as predictors of rubella antibody titers and fatigue - a prospective double blind study. J Psychiatr Res 33:389-395 81. Phillips AC, Carroll D, Burns VE et al. (2005) Neuroticism, cortisol reactivity, and antibody response to vaccination. Psychophysiology 42:232-238

82. Marsland AL, Cohen S, Rabin BS et al. (2006) Trait positive affect and antibody response to hepatitis B vaccination. Brain Behav Immun 20:261-269. doi:S0889-1591(05)00139-X [pii]10.1016/j.bbi.2005.08.009

83. Kohut ML, Cooper MM, Nickolaus MS et al. (2002) Exercise and psychosocial factors modulate immunity to influenza vaccine in elderly individuals. J Gerontol 57:557-562 84. Vedhara K, Bennett PD, Clark S et al. (2003) Enhancement of antibody responses to influenza vaccination in the elderly following a cognitive-behavioural stress management intervention. Psychother Psychosom 72:245-252 